\title{
Postpartum Emotional Psychopathological Outcomes
}

\author{
Javier Quintero*, Sonia Fernandez-Rojo, Ester Chapela, Miriam Felix and Fernando Mora \\ Hospital Universitario Infanta Leonor, Madrid, 28031 Spain \\ *Corresponding author: Javier Quintero, MD, Ph.D, Hospital Universitario Infanta Leonor, Madrid, 28031 Spain, Tel: +341918952; E-mail: \\ fjquinterog@salud.madrid.org
}

Received date: May 19, 2014, Accepted date: May 27, 2014, Published date: May 31, 2014

Copyright: ( 2014 Quintero J, et al. This is an open-access article distributed under the terms of the Creative Commons Attribution License, which permits unrestricted use, distribution, and reproduction in any medium, provided the original author and source are credited.

\begin{abstract}
Pregnancy and birth can cause emotional transformation. It is a period of adaptation for biological, emotional and psychological changes. The relation between maternity and mood disorders are well described. The most studied emotional problems related to childbirth are the maternity or baby blues, the most common, and the least severe. The interest for baby blues started in 1950's when Moloney described a syndrome. Also the Postpartum depression, is an episode of depression that occurs in women shortly after childbirth. And the puerperal psychosis that is a severe psychiatric disorder. There is little evidence to explain the pathogenesis of these conditions. These emotional outcomes, can also affect the attachment development of the newborn, and can cause repercussion in child latter life.

The emotional outcomes during postpartum, should be considered and evaluated by the clinicians, as the prevalence and risk for emotional problems during this period is high. Prevention and early intervention strategies could minimize the influence of postpartum maternal and emotional distress and also infant developmental outcomes.
\end{abstract}

Keywords: Babyblues; Postpartum depression; Postpartum psychosis; Prevention depression; Puerperal psychosis; Children attachment

\section{Introduction}

Pregnancy is a beautiful moment in the woman's life, and should be safeguarded. Pregnancy and birth can cause emotional transformation, bliss and happiness that can affect women. It is a period of adaptation with biological, emotional and psychological changes that in most cases occur without any trouble. As specialist, we should focus on any special issues that could raise some risk, not only for the women health, but also for the baby. Mental health is an integral part of health and a state of wellbeing [1].

The relation between maternity and mood disorders are well described in the History of medicine. During pregnancy and mainly the postpartum some biochemical, hormonal, psychological and social changes take place that can lead to an increment of women vulnerability such as mood disorders.

During the childbirth women can be at high risk of mental health impairment, some describes it as common and others specific for episodes in life. In a recent study Glasheenet cols [2] studied the prevalence of past month serious psychological distress in woman and the prevalence was $6.4 \%$ in first trimester of pregnancy and $3.9 \%$ in third. In postpartum women, the prevalence was ranged from $4.6 \%$ to $6.9 \%$, but more interesting over half of pregnant and postpartum women with past month serious psychological distress were not receiving any mental health treatment [2]. These conditions affect any cultural or economic status, but the risk of non-psychotic perinatal mental disorders seems to be more prevalent in lower-middle-income countries, particularly among poorer women with gender-based risks or a psychiatric history [3].
The most observed emotional problems related to childbirth are probably the maternity or babyblues, the most common, and the least severe, postpartum depression the best studied, and the puerperal psychosis, the most severe and clear psychiatric emergency. These emotional outcomes, can also affect the attachment development of the newborn [4], and can cause repercussion in child latter life. Prevention and early intervention strategies would benefit from an understanding of the influence of postpartum maternal and emotional distress on a broader spectrum of infant developmental outcomes [5]. This article reviews these 3 clinical pictures.

\section{Baby Blues}

Baby blues also called maternity blues or postpartum blues, is a common mild affective syndrome that could be experienced by women after delivery. Scientific interest for baby blues started in 1950's when Moloney described a syndrome, which labeled "third day depression", as a mild depressive reaction that involved fatigue, despondency, tearfulness and difficulty thinking clearly [6]. Many symptomatic descriptions have been made ever since on Baby Blues [7-10]. However, this battery of symptoms is not considered as a clinical diagnosis by the Psychiatric Diagnostic and Statistical Manual of Mental Disorders, fourth edition, text revision (DSM-IV-TR) [11] or the International Classification of Diseases, $10^{\text {th }}$ revision [12].

The characteristic symptoms comprise emotional lability, weeping, anxiety, fatigue, insomnia, irritability, mood or elation and cognitive difficulty including poor attention, distractibility and poor recent memory [7-9]. Emotional lability is the most prominent symptom of the maternity blues [8,9] with mood swings that might take place several times a day. Likewise, depression or sadness do not seem to be typical of the blues [9], in fact, when women weep do not mean that she feel depressed [13]. 
Maternity blues onset commence in the first few days after delivery, increased and finally remit spontaneously after seven to ten $[8,9]$ days. Several studies have described symptoms peaks on different days during the first week: day one [6], day three [14], day five [15-17] or between days four and six [18]. Women who do not improve in two weeks should be evaluated for a postpartum depression, a more serious disorder.

This syndrome is considered to be very common; nevertheless the range of estimated prevalence is quite wide, from $1.8 \%$ [19] to $80 \%$ [20]. This variability is due to the lack of operational criteria for the diagnosis [9], sample differences and the variation in timing and number of assessments [8]. Despite the variability of results, it seems to be a trend to find a higher prevalence in studies carried out in Westerns countries [9].

Several factors have been related to Baby Blues. The postpartum is a period when important hormonal changes take place and furthermore, the mother must adapt to a new life with more demands and responsibilities. As a result, biological, psychological and social factors have been hypothesized as relevant in baby blues causation, nevertheless, study results differ widely even holding opposite conclusions. Rondon [9] concludes that baby blues causation is mostly biological and states that there are no clear relationships with demographic of psychological factors, while Henshaw [8] states the opposite biological correlations are inconclusive and there are convincing relationship between maternity blues and some psychological variables such as a past history of depression or neuroticism.

It is unknown to some extent that baby blues may be related to postpartum biological changes. Several studies report an association between the blues and the abrupt withdrawal of estrogen and progesterone levels after delivery [21,22]. Others found no association with progesterone concentrations $[23,24]$. At the same time, few studies report an association between cortisol levels and mood in the early puerperium [21]. Other studies suggested that the blues may be related to reduced plasma tryptophan during the early postpartum period [25]. Finally, while some studies suggest that thyroid dysfunction might be associated with the development of maternity blues [26], others report no differences in respect to thyroid hormones between women with and without blues [21].

Many variables have been analyzed regarding psychosocial risk factors: sociodemographic and obstetric factors, infant feeding and care, anxiety and mood swings during pregnancy, personality, life events, social support and previous mood disorder [8]; nevertheless the findings are not always conclusive.

In regard to sociodemographic risk factors, no relationship has been found between baby blues and social class, age [27], occupation or socioeconomic level [28]. Low educational level has been related to maternity blues by some authors [28] but not by others [27]. Some studies have found a relationship between being a single mother and baby blues [16,29]; however, others failed to show this relationship [28].

Obstetric factors, pregnancy complications [28] and admissions during pregnancy [16] have been related to postpartum blues. There is no agreement on caesarean delivery, Gonadikis et al. [30] found a significant relationship with baby blues, whereas Kosińska-Kaczyńska et al. found no association [28].
There are reports of association with past history of depression [28]; with reference to anxiety and mood during pregnancy, baby blues have been related to depressive feelings the last month prior to delivery [30], anxiety on the day of delivery [30] and mood change during pregnancy [16]. Finally, stressful events during pregnancy [30] and the lack of family support [28] have also been related to maternity blues.

An interesting question is the relation between maternity blues and postpartum depression. As detailed above, sadness is not a typical symptom of the baby blues while it is a core symptom in postpartum depression; many authors have argued that depression is not part of the blues spectrum [9,31]. At the same time, it should be pointed out that several researchers consider the blues as a well-established risk factor for postpartum depression [8,32-34]. Some studies have shown a link between retrospective recall of blues symptoms and depression later in the postpartum period [35] prospective studies have also demonstrated a relationship [36,37]. Nevertheless others found no association between blues and postpartum depression [38]. However, there is no clear set of clues, presently to predict which women reporting maternity blues will present depression [9] as a result, these women should be carefully observed during a period of time by their practitioner or midwife in order to identify a potential postpartum depression as soon as possible.

The baby blues episodes are mild, time-limited and resolve spontaneously within the first two postpartum weeks. Specific treatment is not necessary. However, given its high prevalence and its potential risk of developing postpartum depression disorder it is important to establish some prevention measures. Firstly, pregnant women should be educated about postpartum blues in order to foster an appropriate understanding and coping with these emotional changes. Secondly, women should be encouraged to inform their physician or midwives about their blues experience, especially if their symptoms last more than two weeks. Thirdly, screening measures for early postpartum affective symptoms should be implemented as routine in primary care in order to detect women at risk in case they did not look for help. Finally, women with maternity blues should be carefully observed in the first weeks postpartum with the aim to identify those at risk of developing postpartum depression and providing treatment at an early stage of the disorder.

\section{Postpartum Depression}

The term puerperal or postpartum depression refers to an episode of depression that occurs in women shortly after childbirth [39]. Childbirth is considered a stressful life event and, for many authors, the postpartum is a high-risk period for the development of affective disorders [40]. Between 3-6\% of women will experience the onset of a major depressive episode during the weeks or months after delivery [41], some authors raise the incidence to $15 \%$ [42]. About $50 \%$ of episodes of postpartum depression begin before birth; therefore, it would be wise to define them as peripartum episodes [41]. In women with a depressive episode after a pregnancy, the risk of recurrence may be as high as 50 to $62 \%$ [43].

There is little evidence to explain the pathogenesis of this condition. Regarding hormonal changes [44], the hypothesis of greater sensitivity to neuromodulator action of estrogen and progesterone in the CNS is managed. It has also been related to genetic factors [45] and major life events [46]. Other factors involved such as obstetric complications, mode of delivery and type of feeding (breastfeeding or not), are all inconsistent and sometimes reveal contradictory results $[47,48]$. 


\begin{tabular}{|l|l|}
\hline \multicolumn{2}{|l|}{ Risk factors } \\
\hline $\begin{array}{l}\text { Past history of depression or bipolar } \\
\text { disorder. }\end{array}$ & Lack of social and financial support. \\
\hline Past history of postpartum depression. & Doubts about pregnancy. \\
\hline Family history of psychiatric disorders. & Intimate partner violence. \\
\hline History of physical or sexual abuse. & Lack of social and financial support. \\
\hline $\begin{array}{l}\text { Current subsyndromal depressive } \\
\text { symptoms. }\end{array}$ & Pregestational or gestational diabetes. \\
\hline Young age. & Congenital malformation in the infant. \\
\hline Stressful life events. & $\begin{array}{l}\text { Childcare-related stressors such as } \\
\text { inconsolable infant crying. }\end{array}$ \\
\hline & $\begin{array}{l}\text { Personality traits (high neuroticism and } \\
\text { high introversion). }\end{array}$ \\
\hline
\end{tabular}

Table 1: Risk factors [49].

It generally begins with typical symptoms of an alteration of physiological functions such as sleep or appetite, lack of energy or libido [49]. These symptoms may mask the diagnosis as they could be explained in the context of a normal puerperium. Lack of sleep and fatigue secondary to neonatal care are part of postnatal normal care; however, patients with an inability to sleep at times or with sustained lack of appetite may have postpartum depression [50].

Moreover, the onset may be similar to that of non-puerperal major depression. Nevertheless, in postpartum depression often predominates the crying and emotional lability, guilty thoughts related to maternity and disability, irritability and ruminations about harming oneself or the baby (described as "scary thoughts", are generally experienced as unacceptable). Clear rejection of the baby can occur in the most severe cases. Symptoms concerning somatic functions are often atypical (hypersomnia and increased appetite) [39].

The criteria for the diagnosis of postpartum depression are the same as those of a nonpostpartum major depressive episode, but indicating the onset within a period of time following birth of a live child:

- For mood "episodes that are associated with the puerperium" the World Health Organization's International Classification of Diseases-10th Revision (ICD-10) requires onset of the episode within six weeks of delivery.

- In the American Psychiatric Association's Diagnostic and Statistical Manual, Fifth Edition (DSM-5) the diagnosis of depression during the postpartum period still utilizes the onset specifier format. However, the specifier has changed, it is now titled "with peripartum onset" which is defined as the most recent episode occurring during pregnancy as well as in the four weeks following delivery [41].

General considerations before treatment [39]:

- A complete medical history including physical examinations is necessary in order to make sure that symptoms are not due to the direct physiological effects of a substance or a general condition (e.g. hypothyroidism usually occurs in $10 \%$ of women after childbirth).

- Treatment should be initiated when symptoms cause clinically significant distress or impairment in social or occupational functioning, when suicidal thoughts appear as well as behavioral problems, neglect of baby or personal care, severe insomnia and high levels of anxiety.

- The kind of symptoms (agitation, anxiety, insomnia, psychomotor retardation or / obsessions) may define the type of treatment to be chosen.

- Consider stopping breastfeeding when pharmacological treatment is prescribed, there is always the possibility that small amounts of medications can be found in milk. It should be interrupted in preterm baby or liver failure.

- When breastfeeding is maintained, treatment should be administered just after food intake and wait for a while before putting the infant to sleep. It is recommended to monitor the adverse effects on mother and baby (sedation, irritability, sleeping and eating patterns changes).

\section{Psychopharmacological Options}

\section{Antidepressants}

The antidepressant efficacy is similar to that expected in nonpospartum depressive episode. In those cases where breastfeeding is maintained possible risks to infants must be taken into account because antidepressants can be secreted into breast milk [51]. Selective serotonin reuptake inhibitors (SSRIs), serotonin-norepinephrine reuptake inhibitors (SNRSs) and atypical antidepressants concentrations are generally low.

- SSRIs: infant serum concentrations appear to be lower with paroxetine and sertraline and higher with citalopram, fluoxetine [52].

- SNRs: venlafaxine, desvenlafaxine and duloxetine appear to be safe to use in breastfeeding women [53]. However, infant serum concentrations of venlafaxine and desvenlafaxine are often detectable.

- Atypical antidepressants have been studied in few nursing infants. Little information is available about the use of agomelatine in lactating women. Infant exposure to bupropion through breast milk may be low [54]. Infant serum concentrations of mirtazapine are generally below the limit of detection [55].

\section{Benzodiazepines}

Benzodiazepines should be avoided. Although they can be found in breast milk and infant serum, an occasional administration is not contraindicated in breastfeeding, but must be consider very carefully before prescribing and counselling of breastfeeding [56]. Administration of low doses of drugs that have no active metabolites such as lorazepam [57] could be occasionally used. Diazepam is not compatible with breastfeeding due to its high concentrations in infant serum [58].

\section{Mood estabilizers}

Lithium is contraindicated during lactation because breast milk and infant serum concentrations are frequently substantial, with infant levels of 10 to $50 \%$ of maternal serum concentrations and the subsequent risk of toxic symptoms (cyanosis, hypothermia, hypotonia) $[39,59]$. Carbamazepine and valproate are compatible with breastfeeding because breast milk concentrations are generally low or undetectable [57]. In the case of carbamazepine treatment is 
recommended to control liver enzymes and blood counts in the infant [56].

Electroconvulsive Therapy, (ETC) is a safe treatment for postpartum depression with few adverse effects for either the mother or infant [60].

- For severe postpartum major depression that may not respond to pharmacotherapy (patients refractory to multiple medication trials) [61].

- When rapid treatment is necessary (psychotic features, suicide or infanticide risk).

- For patients with severe postpartum major depression who are breastfeeding, because of its low adverse effects upon lactation [62].

Psychotherapy combined with antidepressants are more effective than given separately; both cognitive behavioral therapy (based on the theory of learning and correcting cognitive distortions) and interpersonal therapy (where it gets more emphasis on interpersonal momentary issues) are the most effective in this type of depression. Psychotherapy is suggested for mild to moderate, postpartum unipolar major depression [63]. This approach is especially useful for lactating patients who want to avoid neonatal exposure to antidepressants. However, pharmacotherapy is a reasonable alternative if psychotherapy is not successful, declined not available, or if the patient has previously responded to antidepressants [49].

In patients with high risk of developing a postpartum depression (due to a prior episode) some authors suggested prophylaxis with antidepressants even during the third term of pregnancy. If there is history of successful treatment of the previous episode, prophylaxis should be recommended with the same antidepressant. Interpersonal psychotherapy or psychosocial interventions are also advocated. Nevertheless, pharmacotherapy is more widely available than psychotherapy for prevention [49]. Counselling of breastfeeding mothers should be carefully assessed.

\section{Postpartum or Puerperal Psychosis}

The term puerperal or postpartum psychosis refers to an episode of acute psychosis that occurs in mothers shortly after childbirth. The main difference from the rest of puerperal psychiatric disorders [64] is the presence of psychotic symptoms, meaning a disturbance in perception of reality manifested by delusions, hallucinations or thought disorganization.

The typical clinical condition is characterized by the debut in the early postpartum weeks of acute delusional symptoms combined with confusion and mood manifestations, with a fluctuating course. It can occur in women with or without previous psychiatric illness. Clinical evidence and genetic studies support the hypothesis that puerperal psychosis is a manifestation of bipolar disorder that is precipitated by the postnatal period [65].

Puerperal psychosis occurs in 1-2 out of 1000 births in general population. The incidence is higher in case of personal or family history of mood disorders, especially bipolar disorder type I, in which the frequency increases to $20-25 \%$ [66]. After an episode of puerperal psychosis risk of recurrence in future deliveries is estimate at $30 \%$. Other risk factors described include primiparity, caesarean delivery and recent discontinuation of lithium or other mood stabilizers. The debut is commonly abrupt within the first month following childbirth ( $80 \%$ of cases), often within the first week (40\%) [50].
The aetiology of postpartum psychosis remains unknown. Most researches are focus on genetic and hormonal factors. Psychosocial factors seem to be more decisive in case of maternity blues and puerperal depression [67].

- Genetic factors: Family studies of women with one or more episodes have found an aggregation of bipolar and other mood disorders.

- Biological factors: there are many hypotheses relating puerperal psychosis with the hormonal changes that occur in the postpartum period, none of them have been confirmed so far. These theories are based on rapid hormonal changes in levels of progesterone and/or estrogens, thyroid axis, gonadotropin prolactin or corticosteroids [68].

Psychosocial factors: particularly the changes in family role derived from the arrival of a child. In this sense, the immature personality could predispose to postpartum psychiatric disorders.

Puerperal psychosis can manifest as maniac or mixed episodes, severe depressive episodes with psychotic symptoms, typical schizophrenic episodes or as an acute delusional psychosis with differential characteristics. The key feature that differentiates puerperal psychosis from other postpartum psychiatric disorders is the appearance of psychotic symptoms with loss of contact with reality, accompanied by confusion, perplexity and emotional lability. There are typically affective symptoms with manic or depressed mood, severe insomnia, rapid mood swings, anxiety, irritability, and psychomotor agitation. Symptoms begin abruptly within the first 3 weeks postpartum, with a peak frequency on the day tenth. The syndrome based on acute delusional psychosis, commonly preceded by prodromal symptoms such as anxiety, fatigue, depressed mood and sleep disturbances. Persistent severe insomnia (not related to caring for the newborn) is often the first manifestation [69]. The clinical presentation includes rapid mood swings, delusions, cognitive symptoms, psychomotor disorders, sleep disturbances and hallucinations. The mood may be elevated, mixed or depressed, and is often rapid changing and typically accompanied by mood lability and confusion. The patient's mental state may rapidly change from hyperactivity to stupor, from verbiage to silence. Specific delusions are related to the baby, with mother believing that baby is devil or the reincarnation of a deity, that the child is dead or has been replaced or changed sex, or being convinced that someone is trying to damage or kidnap the baby. Substantial decrease in hour of sleep and cognitive disturbances, including confusion and fluctuations in level of consciousness or even disorientation to person, place or time, without evidence of other causes of delirium. Hallucinations may be present, sometimes in the form of command auditory hallucinations instructing the mother to harm the baby or herself.

Puerperal psychosis is a severe disorder with high risk of behaviour disturbances and mortality (5\% complete suicide and $4 \%$ infanticide) [70]. The natural evolution of the episode is the gradual and spontaneous disappearance of symptoms over weeks or months. The other psychotic disorders that may manifest in postpartum period include major depressive disorder with psychotic symptoms, schizoaffective disorder, schizophrenia and brief psychotic disorder.

The diagnosis of puerperal psychosis should be considered when an episode of psychotic symptoms occurs in temporal relation to childbirth. Medical evaluation should include clinical history, physical and neurologic examination and laboratory test to exclude medical 
and toxicological causes. The mental examination need to focus on the onset and course of psychotic symptoms, features of mood disturbances, functional and behavioural repercussion, personal and family history of psychiatric disorders, asses safety of baby and mother and comorbid substance use disorder [50].

The DSM-5 [41] do not consider puerperal psychosis as a distinct diagnosis, therefore the episode should be classified considering the mood or psychotic disorder plus the specified "with postpartum onset" whenever symptoms begin during pregnancy or within 4 weeks postpartum. The ICD-10 prolongs the postpartum period until 6 weeks postpartum. Diagnosis of underlying psychiatric disorder:

Puerperal psychosis is believed a manifestation of a bipolar disorder [66], especially type I, with many relative evidences among them:

- Postpartum psychosis manifest as a manic, depressive or mixed episode among patients with bipolar disorder already diagnosed.

- Women with bipolar disorder are at high risk of recurrence in pregnancy and postpartum, which may present as postpartum psychosis.

- Women who discontinue mood stabilizers before or during the pregnancy may be at an increased risk of postpartum psychosis.

- For women with no prior history, puerperal psychosis may be the first manifestation of bipolar disorder.

The differential diagnosis must be established early with the rest of puerperal psychiatric disorders by exploring the existence of psychotic symptoms. Substance use disorders may cause psychosis in postpartum period as the result of abrupt withdrawal or intoxication. Medical causes of psychosis need to be excluded, as well as medical comorbid conditions that may exacerbate psychosis: metabolic encephalopathy, endocrine disorders (e.g., thyroiditis), neurologic events (e.g., seizures, neoplasms, trauma), etc.

Acute management of puerperal psychosis starts with ensuring safety of both the patient and the baby and initiating pharmacotherapy. Next steps include medical evaluation to exclude other potential causes as well as diagnosing and treating the underlying psychiatric disorder [65]. Postpartum psychosis is considered a psychiatric emergency that usually requires hospitalization. The mother should not be left alone with the infant, some authors recommend mother-infant specific units, but not many countries have them. During hospitalization supervised day-time visits with infants in order to prevent attachment disturbance can be carried out.

Pharmacological management is basically similar to other psychotic conditions besides postpartum period, with antipsychotic medications as the first choice drugs [65]. As far as we know there are no controlled trials, but randomized that have demonstrated efficacy in reducing psychosis [71]. Some authors warn off an increased risk of neuroleptic malignant syndrome in postpartum episodes, while others consider there is no increased rate of adverse effects from antipsychotics in these patients.

Atypical antipsychotics should be favoured over the typical antipsychotics because of their mood stabilizing properties and lower risk of extrapyramidal symptoms and tardive dyskinesia. If conventional antipsychotic medications are used, the high-potency agents, such as haloperidol, perphenazine, trifluoperazine, and thiothixene are usually recommended. Mood stabilizers may be also considered, especially considering that many cases are due to an underling bipolar disorder [65]. Other adjunctive medications as benzodiazepines can be effective to manage sleep disturbances, anxiety and agitation. Patients should be treated to remission and should maintain medication for at least one year.

Some women with mild postpartum psychosis may continue breastfeeding, after exhaustive evaluation of risk/benefit, but women should not be left alone with the child at any time. Considering that all psychotropic medications are transferred into breast milk and pass on to the child, possible benefits need to be carefully considered against risks for each drug. In case of breastfeeding, select medications with better safety data should be considered in pregnancy and lactation [72]. Especial caution is to be taken using bromocriptine to suppress lactation, because it has been associated with the induction of psychotic symptoms. Counselling of breastfeeding mothers should be carefully assessed in these circumstances.

Electroconvulsive therapy is also considered a treatment of choice due to rapid action and less interferences with breastfeeding, even when there is no evidence that electroconvulsive therapy is better than pharmacotherapy [73]. There is lack of randomized trials of electroconvulsive therapy for puerperal psychosis, while randomized trials in non-puerperal affective episodes show better results in depression with psychotic symptoms than in mania. Some authors recommend electroconvulsive therapy just in case of resistance or ill tolerance to medication. The number of sessions depends on clinical evolution. Risk of thrombotic complications should be considered within the first four weeks postpartum [73].

During acute puerperal psychosis family should be advised to take care of the newborn and prevent mother from being alone with baby. Most patients require admission to prevent risk of self and/or baby harm. Psychoeducational interventions are important to prevent relapse and help future family planning. Patients and relatives should receive information about risks and early symptoms of recurrence.

The evolution of acute episode is favourable in $70-80 \%$ cases. Good prognostic factors include the absence of psychiatric history, early abrupt onset and the presence of affective symptoms. The acute clinical condition usually improves with medication or electroconvulsive therapy, but early relapses are frequent, occasionally, turning to mixed or depressive states. Postpartum psychosis shows high risk of recurrence. Long-term studies indicate that $51-65 \%$ will present one or more episodes of mood disturbance unrelated to postpartum throughout lifetime [74].

During medical care of pregnancy or postpartum period, few standard screening questions should be considered: "Have you ever had any periods of increased energy lasting at least four days? Were they accompanied by elevated or grandiose mood, decreased need for sleep, rapid speech, racing thoughts, or unusual creativity and productivity? Do you have family history of bipolar disorder?". Women screening positive should be monitored in pospartum period and receive a more detailed psychiatric evaluation. Women with bipolar disorder or previous postpartum psychosis may receive treatment prophylactically even during pregnancy and in postpartum period [75].

\section{Conclusions}

The emotional outcomes during postpartum, should be evaluated and considered by the clinicians, as the prevalence and risk for problems even disorders during this period is high. Most of the cases 
are punctual and adjustment procedures, the "baby blues", but there are other problems that can have negative impact on the mother and also on the baby. Special interest should be focused on the postpartum depression and mainly the postpartum psychosis, as described.

Recommendations were generally consistent on the need of evaluating the mood after birth. The most extended and validated scale for this evaluation is the Edinburgh Postnatal Depression Scale [76] but the suggested time for the routine evaluation is not clear [77]. The use of rigorously developed, evidence-based guidelines has the potential to improve patient care. As far as we know, there are not many guidelines and/or consensus and the majority has been developed in the United States of America or the United Kingdom, therefore, bias and more specifically region and cultural guidelines [9] should be considered [77].

In some cases the pharmacological treatment is needed, hence counselling of breastfeeding mothers should be carefully assessed. Pharmacokinetic drug characteristics, disease severity, behavioural or psychosocial alternatives, preventative interventions and possible impact of discontinuing breastfeeding on the maternal-infant relationship should all be taken into account [58].

From our point of view, those disorders are important enough not only in terms of maternal view, but also in the baby relationship building, and crucial in the child attachment [4]. Because of this double repercussion the emotional outcome after birth, should be considered and evaluated as routine, and the health workers, like midwives, nurses, practitioners, should know how to detect the relative problems, and how seek for specialized help.

Prevention and early intervention strategies on the emotional outcomes after pregnancy, would benefit the mother health, and also would benefit the infant developmental outcomes [5]. All those aspect should be analyzed and individualized carefully for each patient conditions, and physician expertise.

\section{References}

1. WHO (World Health Organization) (1946). Official Records of the World Health Organization, № 2, Nueva York.

2. Glasheen C, Colpe L, Hoffman V, Warren LK (2014) Prevalence of Serious Psychological Distress and Mental Health Treatment in a National Sample of Pregnant and Postpartum Women. Matern Child Health J .

3. Fisher J, Cabral de Mello M, Patel V, Rahman A, Tran T, et al. (2012) Prevalence and determinants of common perinatal mental disorders in women in low- and lower-middle-income countries: a systematic review. Bull World Health Organ 90: 139G-149G.

4. Kingston D, Tough S, Whitfield H (2012) Prenatal and postpartum maternal psychological distress and infant development: a systematic review. Child Psychiatry Hum Dev 43: 683-714.

5. Gunlicks ML, Weissman MM (2008) Change in child psychopathology with improvement in parental depression: a systematic review. J Am Acad Child Adolesc Psychiatry 47: 379-389.

6. Moloney J (1952) Postpartum depression or third day depression following childbirth. New Orleans Child Parent Digest 6: 20-32.

7. Gale S, Harlow BL (2003) Postpartum mood disorders: a review of clinical and epidemiological factors. J Psychosom Obstet Gynaecol 24: 257-266.

8. Henshaw C (2003) Mood disturbance in the early puerperium: a review. Arch Womens Ment Health 6 Suppl 2: S33-42.

9. Rondon MB (2003) Maternity blues: cross-cultural variations and emotional changes. PsychiatUpdat 10: 167-171.
10. O'Hara MW, Schlechte JA, Lewis DA, Wright EJ (1991) Prospective study of postpartum blues. Biologic and psychosocial factors. Arch Gen Psychiatry 48: 801-806.

11. American Psychiatric Association (2000) Diagnostic and Statistical Manual of Mental Disorders, (Fourth Edn), American Medical Assoc, Washington, DC.

12. World Health Organization (2004) International Statistical Classification of Diseases and Related Health Problems, (Tenth Edn), Geneva: World Health Organization.

13. Yalom ID, Lunde DT, Moos RH, Hamburg DA (1968) "Postpartum blues" syndrome. A description and related variables. Arch Gen Psychiatry 18: 16-27.

14. Condon JT, Watson TL (1987) The maternity blues: exploration of a psychological hypothesis. Acta Psychiatr Scand 76: 164-171.

15. Dowlati Y, Segal ZV, Ravindran AV, Steiner M, Stewart DE, et al. (2014) Effect of dysfunctional attitudes and postpartum state on vulnerability to depressed mood. J Affect Disord 161: 16-20.

16. Adewuya AO (2005) The maternity blues in Western Nigerian women: prevalence and risk factors. Am J Obstet Gynecol 193: 1522-1525.

17. O'Keane V, Lightman S, Patrick, K, Marsh M, Papadopoulos AS, Pawlby S, Seneviratne G, Taylor A, Moore R (2011). Changes in the maternal hypothalamic-pituitary-adrenal axis during the early puerperium may be related to the postpartum 'blues'. J Neuroendocrinol 23: 1149-55.

18. Wilkie G, Shapiro CM (1992) Sleep deprivation and the postnatal blues. J Psychosom Res 36: 309-316.

19. Romero-Gutiérrez G, Dueñas-de la Rosa EM, Regalado-Cedillo CA, Ponce-Ponce de León AL (2010) [Prevalence of maternal sadness and its associated factors]. Ginecol Obstet Mex 78: 53-57.

20. Nonacs R, Cohen LS (1998) Postpartum mood disorders: diagnosis and treatment guidelines. J Clin Psychiatry 59 Suppl 2: 34-40.

21. Okano T, Nomura J (1992) Endocrine study of the maternity blues. Prog Neuropsychopharmacol Biol Psychiatry 16: 921-932.

22. Doornbos B, Fokkema DS, Molhoek M, Tanke MA, Postema F, et al. (2009) Abrupt rather than gradual hormonal changes induce postpartum blues-like behavior in rats. Life Sci 84: 69-74.

23. Chatzicharalampous C, Rizos D, Pliatsika P, Leonardou A, Hasiakos D, et al. (2011) Reproductive hormones and postpartum mood disturbances in Greek women. Gynecol Endocrinol 27: 543-550.

24. Kuevi V, Causon R, Dixson AF, Everard DM, Hall JM, et al. (1983) Plasma amine and hormone changes in 'post-partum blues'. Clin Endocrinol (Oxf) 19: 39-46.

25. Gard PR, Handley SL, Parsons AD, Waldron G (1986) A multivariate investigation of postpartum mood disturbance. Br J Psychiatry 148: 567-575.

26. Ijuin T, Douchi T, Yamamoto S, Ijuin Y, Nagata Y (1998) The relationship between maternity blues and thyroid dysfunction. J Obstet Gynaecol Res 24: 49-55.

27. Yamashita H, Yoshida $\mathrm{K}$ (2003) [Screening and intervention for depressive mothers of new-born infants]. Seishin Shinkeigaku Zasshi 105: 1129-1135.

28. Kosinska-Kaczynska K, Horosz E, Wielgo M, Szymusik I (2008) [Affective disorders in the first week after the delivery: prevalence and risk factors]. Ginekol Pol 79: 182-185.

29. Faisal-Cury A, Menezes PR, Tedesco JJ, Kahalle S, Zugaib M (2008) Maternity "blues": prevalence and risk factors. Span J Psychol 11: 593-599.

30. Gonidakis F, Rabavilas AD, Varsou E, Kreatsas G, Christodoulou GN (2007) Maternity blues in Athens, Greece: a study during the first 3 days after delivery. J Affect Disord 99: 107-115.

31. Kennerley H, Gath D (1989) Maternity blues. I. Detection and measurement by questionnaire. Br J Psychiatry 155: 356-362.

32. Reck C, Stehle E, Reinig K, Mundt C (2009) Maternity blues as a predictor of DSM-IV depression and anxiety disorders in the first three months postpartum. J Affect Disord 113: 77-87. 
33. Stowe ZN, Nemeroff CB (1995) Women at risk for postpartum-onset major depression. Am J Obstet Gynecol 173: 639-645.

34. Ferretti F, Franca A, Folin M (2013) [Risk factors associated with postnatal depressive symptomatology: a study conducted in the Southern Area of the Local Health Unit of Modena]. Epidemiol Prev 37: 138-144.

35. Sharp D (1993) Childbirth-related emotional disorders: a longitudinal prospective study in primary care.

36. Fossey L, Papiernik E, Bydlowski M (1997) Postpartum blues: a clinical syndrome and predictor of postnatal depression? J Psychosom Obstet Gynaecol 18: 17-21.

37. Ishikawa N, Goto S, Murase S, Kanai A, Masuda T, et al. (2011) Prospective study of maternal depressive symptomatology among Japanese women. J Psychosom Res 71: 264-269.

38. Kennerley H, Gath D (1989) Maternity blues. III. Associations with obstetric, psychological, and psychiatric factors. Br J Psychiatry 155: 367-373.

39. Leal C (1999) Trastornos depresivos en la mujer. Masson S.A.

40. Bebbington PE, Dean C, Der G, Hurry J, Tennant C (1991) Gender, parity and the prevalence of minor affective disorder. Br J Psychiatry 158: 40-45.

41. American Psychiatric Association (2013) Diagnostic and statistical manual of mental disorders (5th edn) VA: American Psychiatric Publishing, Arlington.

42. Wisner KL, Chambers C, Sit DK (2006) Postpartum depression: a major public health problem. JAMA 296: 2616-2618.

43. Altshuler LL, Hendrick V, Cohen LS (1998) Course of mood and anxiety disorders during pregnancy and the postpartum period. J Clin Psychiatry 59: 29-33.

44. Bloch M, Schmidt PJ, Danaceau M, Murphy J, Nieman L, et al. (2000) Effects of gonadal steroids in women with a history of postpartum depression. Am J Psychiatry 157: 924-930.

45. Treloar SA, Martin NG, Bucholz KK, Madden PA, Heath AC (1999) Genetic influences on post-natal depressive symptoms: findings from an Australian twin sample. Psychol Med 29: 645-654.

46. Wilson LM, Reid AJ, Midmer DK, Biringer A, Carroll JC, et al. (1996) Antenatal psychosocial risk factors associated with adverse postpartum family outcomes. CMAJ 154: 785-799.

47. Warner R, Appleby L, Whitton A, Faragher B (1999) Demographic and obstetric risk factors for postnatal year. Br J Psychiatry 144: 453-462.

48. O'Hara MW, Schlechte JA, Lewis DA, Varner MW (1991) Controlled prospective study of postpartum mood disorders: psychological, environmental, and hormonal variables. J Abnorm Psychol 100: 63-73.

49. Roy-Byrne PP (2014) Postpartum blues and unipolar depression: Epidemiology, clinical features, assesment and diagnosis. Uptodate.

50. Nonacs R, Cohen LS (1998) Postpartum mood disorders: diagnosis and treatment guidelines. J Clin Psychiatry 59 Suppl 2: 34-40.

51. Berle JO, Spigset O (2011) Antidepressant Use During Breastfeeding. Curr Womens Health Rev 7: 28-34.

52. Lam RW, Kennedy SH, Grigoriadis S, McIntyre RS, Milev R, et al. (2009) Canadian Network for Mood and Anxiety Treatments (CANMAT) clinical guidelines for the management of major depressive disorder in adults. III. Pharmacotherapy. J Affect Disord 1: S26.

53. Ilett KF, Hackett LP, Dusci LJ, Roberts MJ, Kristensen JH, et al. (1998) Distribution and excretion of venlafaxine and O-desmethylvenlafaxine in human milk. Br J Clin Pharmacol 45: 459-462.

54. Davis MF, Miller HS, Nolan PE Jr (2009) Bupropion levels in breast milk for 4 mother-infant pairs: more answers to lingering questions. J Clin Psychiatry 70: 297-298.
55. Klier CM, Mossaheb N, Lee A, Zernig G (2007) Mirtazapine and breastfeeding: maternal and infant plasma levels. Am J Psychiatry 164: 348-349.

56. Chinchilla A (2013) Tratamientos psiquiátricos. Ediveramerica.

57. Eberhard-Gran M, Eskild A, Opjordsmoen S (2006) Use of psychotropic medications in treating mood disorders during lactation : practical recommendations. CNS Drugs 20: 187-198.

58. Sachs HC; Committee On Drugs (2013) The transfer of drugs and therapeutics into human breast milk: an update on selected topics. Pediatrics 132: e796-809.

59. Grandjean EM, Aubry JM (2009) Lithium: updated human knowledge using an evidence-based approach: part III: clinical safety. CNS Drugs 23:397.

60. Anderson EL, Reti IM (2009) ECT in pregnancy: a review of the literature from 1941 to 2007. Psychosom Med 71: 235-242.

61. UK ECT Review Group (2003) Efficacy and safety of electroconvulsive therapy in depressive disorders: a systematic review and meta-analysis. Lancet 361: 799-808.

62. Jaffe $\mathrm{R}$ (2002) The Practice of Electroconvulsive Therapy: Recommendations for Treatment, Training and Privileging: A Task Force Report of the American Psychiatric Association. 2nd ed. 159: 331.

63. Lumley J, Austin MP, Mitchell C (2004) Intervening to reduce depression after birth: a systematic review of the randomized trials. Int J Technol Assess Health Care 20: 128-144.

64. Gómez Alcalde M (2002) Patología psiquiátrica en el puerperio. Psicosis puerperal. Revista de Neuropsiquiatría; 65: 32-46.

65. Sharma V, Burt VK, Ritchie HL (2010) Assessment and treatment of bipolar II postpartum depression: a review. J Affect Disord 125: 18-26.

66. Chaudron LH, Pies RW (2003) The relationship between postpartum psychosis and bipolar disorder: a review. J Clin Psychiatry 64: 1284-1292.

67. Pfuhlmann B, Stoeber G, Beckmann H (2002) Postpartum psychoses: prognosis, risk factors, and treatment. Curr Psychiatry Rep 4: 185-190.

68. Gentile S (2005) The role of estrogen therapy in postpartum psychiatric disorders: an update. CNS Spectr 10: 944-952.

69. Spinelli MG (2009) Postpartum psychosis: detection of risk and management. Am J Psychiatry 166: 405-408.

70. Spinelli MG (2004) Maternal infanticide associated with mental illness: prevention and the promise of saved lives. Am J Psychiatry 161: 1548-1557.

71. Sharma V, Smith A, Mazmanian D (2006) Olanzapine in the prevention of postpartum psychosis and mood episodes in bipolar disorder. Bipolar Disord 8: 400-404.

72. Klinger G, Stahl B, Fusar-Poli P, Merlob P (2013) Antipsychotic drugs and breastfeeding. Pediatr Endocrinol Rev 10: 308-317.

73. Focht A, Kellner CH (2012) Electroconvulsive therapy (ECT) in the treatment of postpartum psychosis. J ECT 28: 31-33.

74. McGrath L, Peters S, Wieck A, Wittkowski A1 (2013) The process of recovery in women who experienced psychosis following childbirth. BMC Psychiatry 13: 341.

75. Bergink V, Bouvy PF, Vervoort JS, Koorengevel KM, Steegers EA, et al. (2012) Prevention of postpartum psychosis and mania in women at high risk. Am J Psychiatry 169: 609-615.

76. Garcia-Esteve L, Ascaso C, Ojuel J, Navarro P (2003) Validation of the Edinburgh Postnatal Depression Scale (EPDS) in Spanish mothers. J Affect Disord 75: 71-76.

77. Haran C, van Driel M, Mitchell BL, Brodribb WE (2014) Clinical guidelines for postpartum women and infants in primary care-a systematic review. BMC Pregnancy Childbirth 14: 51. 